\title{
Neu entdeckte Radiolaritabbaue in Wien. Bedeutung und Nutzung \\ der St. Veiter Klippenzone im Neolithikum
}

\author{
Oliver Schmitsberger \\ Michael Brandl \\ Martin Penz
}

\begin{abstract}
Zusammenfassung
Im Rahmen des Projekts „BergbauLandschaftWien“ wurden 2016 bis 2018 intensive Forschungen zum neolithischen Radiolaritbergbau in der St. Veiter Klippenzone in Wien und dessen direktem Umland durchgeführt. Die Arbeiten umfassten Prospektion im Gelände, Archivrecherche und Aufnahme bzw. Überprüfung von Altfunden sowie eine kleine Flächengrabung am Gemeindeberg. Als besonders vielversprechendes Gebiet stellte sich das Naturschutzgebiet „Lainzer Tiergarten“ heraus, wo bis Ende 2018 insgesamt 15 bislang unbekannte Abbau- bzw. Nutzungsstellen entdeckt wurden. Diese wurden anhand „bergbautypischer" Oberflächenfunde wie artifizielle Trümmerstücke (Abbauschutt), Entrindungsabschläge, precores, Schlagsteine etc. bei gleichzeitigem Fehlen charakteristischer Siedlungsfunde als solche identifiziert. Zudem erfolgten erste Schritte zur Einbindung der Abbaustellen in die umgebende Siedlungslandschaft und Infrastruktur, um die sozioökonomische Dimension des Bergbauphänomens zu erfassen. Die Nutzung des „Wiener Radiolarits“ ist vom Paläolithikum bis in die Bronzezeit belegt, der eigentliche Bergbau dürfte aber überwiegend im Neolithikum umgegangen sein. Als vorläufiges Ergebnis bleibt festzuhalten, dass die Dimensionen der neolithischen Radiolaritgewinnung in Wien bisher völlig unterschätzt wurden. Es handelt sich nicht, wie bislang angenommen, um vereinzelte Befunde, sondern um ein prähistorisches Bergbaugebiet von europäischem Rang und beträchtlicher Ausdehnung - und mit einem enormen Forschungs- und Erkenntnispotential.
\end{abstract}

\section{Schlüsselbegriffe}

Bergbau, Radiolarit, Neolithikum, Wien, Klippenzone, Ressourcenmanagement.

Abstract - Newly Discovered Radiolarite Mining in Vienna. Importance and Use of the St. Veit Klippen Belt in the Neolithic

Within the framework of the project 'BergbauLandschaftWien' extensive research concerning Neolithic mining activities in the St. Veit Klippen Belt was undertaken between 2016 and 2018 in Vienna and the immediate catchment area. These investigations included surveys, archival research and the recording as well as re-evaluation of old collections, and a small-scale excavation at the Gemeindeberg in Vienna. An especially promising area is the nature reserve known as the 'Lainzer Tiergarten', where altogether 15 so-far unknown radiolarite quarrying and workshop areas were detected by the end of 2018. They were identified based on surface finds characteristic for prehistoric quarrying, such as artificial (quarrying) debris, decortification flakes, precores, hammer stones, etc., in combination with the absence of indications for regular settlement activities. Additionally, initial steps towards incorporating the mining landscape into the surrounding settlement and infrastructure were undertaken in order to grasp the socio-economic dimensions of these mining activities. The use of 'Vienna radiolarite' is evidenced from the Palaeolithic to the Bronze Age; the heydays of mining however appear to be a Neolithic phenomenon. Our preliminary results indicate that the scale of Neolithic radiolarite mining in Vienna has been dramatically underestimated. We are not dealing with isolated findings as previously assumed, but, on the contrary, with a prehistoric mining landscape of European rank and significant extent, providing unprecedented research potential.

\section{Keywords}

Mining, radiolarite, Neolithic, Vienna, Klippen Belt, resource management.

\section{Einleitung}

In direkter Entwicklung aus dem Projekt „Gog \& Magog - Die Mammutjägerzeit in Wien"1 wird seit 2016 unter dem Projekttitel „BergbauLandschaftWien“ (BLW) die

1 Schmitsberger, Neugebauer-Maresch 2015.-Schmitsberger, Neugebauer-Maresch 2018a. - Schmitsberger, NeugebauerMARESCH 2018b. 
Erforschung der neolithischen Silizit-Abbaustellen in und um Wien forciert. ${ }^{2}$ Entstanden ist dieses Nachfolgeprojekt aus der Erkenntnis, dass viele der bislang nur allgemein als Hornstein-Artefaktfundplätze bekannten bzw. überhaupt nur in „grauer Literatur" aufscheinenden oder oral tradierten Fundstellen (besonders in der St. Veiter Klippenzone, zum Teil auch in der Flyschzone) in Wirklichkeit im Kontext von prähistorischem Rohmaterial-Abbau stehen. ${ }^{3}$ Das Projekt baut auf jahrelangen Vorarbeiten auf, die einerseits intensive Survey-Tätigkeiten nicht nur in Wien, sondern im gesamten ostösterreichischen Raum, und andererseits Rohmaterialanalysen umfassten, welche speziell die Radiolaritressourcen des Ostalpenraums und östlich angrenzender Gebiete zum Inhalt hatten.

Im Rahmen des Projekts finden nun Geländearbeiten in Form von Prospektion und punktueller Grabung, Literatur- und Archivrecherchen, Aufnahmen der Altfundbestände und eine Kartierung der prähistorischen Landschaft, die sowohl die Bergbaubefunde als auch die Siedlungslandschaft miteinbezieht, statt.

Schließlich ist ein wesentlicher Aspekt der Urgeschichtsforschung die Erfassung von Ressourcen, welche für die prähistorischen Menschen überlebensnotwendig waren. Darunter kommt besonders Rohstoffen für Steingeräte wie Feuerstein, Hornstein oder Radiolarit eine wesentliche Rolle zu. Diese Materialien wurden aufgrund ihrer Härte und ihres Bruchverhaltens seit der frühesten Menschheitsgeschichte für die Herstellung von scharfkantigen Steingeräten wie Schaber, Projektilspitzen, Messer-, Dolch- und Sichelklingen genutzt. Es geht hier um eine Zeitperiode, in welcher solche Steingeräte generell zu den wichtigsten Werkzeugen gehörten, und - nicht zuletzt aufgrund ihrer materialbedingten Erhaltungsfähigkeit - nachweislich eine der häufigsten archäologischen Fundgattungen darstellen. ${ }^{4}$ Deshalb sind sie besonders gut dazu geeignet, fundamentale Aspekte der sozio-ökonomischen Organisation prähistorischer Gesellschaften zu rekonstruieren, insbesondere menschliches Verhalten sowie die Entwicklung und Veränderungen kultureller Netzwerke. Ein umfassender Ansatz zum Verständnis lithischen Ressourcenmanagements erfordert

\footnotetext{
2 Kooperation zwischen OREA (Institut für Orientalische und Europäische Archäologie, Österreichische Akademie der Wissenschaften), Stadtarchäologie Wien und Vienna Lithothek (Institut für Urgeschichte und Historische Archäologie, Universität Wien) sowie weiteren Partnern (Bundesdenkmalamt, Naturhistorisches Museum Wien, Geologische Bundesanstalt).

3 Z. B. Knödelhütte, Kahlenberg-Süd, Roter Berg, Lainzer Tiergarten Fundstelle 1, Hörndlwald, Sterndlwiese etc.

4 Vgl. AndrefsKy 2009, 65.
}

demnach die Einbindung von Lagerstättenkunde, Herkunftsanalysen und techno-typologischer Untersuchungen archäologischer Fundinventare.

Neben der Nutzung von Flussgeröllen beginnt zur Versorgung mit lithischen Rohstoffen bereits sehr früh prähistorischer Bergbau, wie er auch für das Stadtgebiet von Wien nachgewiesen ist. Bisher ist jedoch nur das neolithisch-frühkupferzeitliche „Hornsteinbergwerk“ auf der Antonshöhe in Wien-Mauer (5. Jt. v. Chr.), gerne auch als „Österreichs ältestes Industriedenkmal“ bezeichnet, in der Fachliteratur einigermaßen bekannt gemacht worden. Allerdings war aufgrund der geologischen Bedingungen abzusehen, dass dies nicht die einzige Stelle sein kann, an welcher in prähistorischen Zeiten auf heutigem Wiener Gebiet Hornstein, bzw. vor allem die spezielle Hornstein-Varietät Radiolarit, abgebaut wurde.

\section{Die geologischen Voraussetzungen}

Die St. Veiter Klippenzone (SVK) im Westen bzw. Südwesten Wiens (und bis auf niederösterreichisches Gebiet reichend) ist aus marinen mesozoischen Sedimenten aufgebaut, welche zum Teil hornstein- bzw. radiolaritführend sind. ${ }^{5}$ Vor allem die Rotenberg-Formation (Fm) und die Fasslgraben-Fm sind hier besonders hervorzuheben, wobei nach den gängigen geochronologischen Datierungen die Rotenberg-Fm in den Oberen Jura und die FasslgrabenFm in den Obersten Jura und in die Unterkreide gestellt werden. ${ }^{6}$ Neueste geologisch-mikropaläontologische Forschungen zeigen aber ein weit komplexeres Bild, 7 demnach gehört die Rotenberg-Fm überwiegend in den Mittleren und nur zu einem geringen Teil in den Oberen Jura, während sich die traditionell älter als erstere eingestufte Hohenauer Wiese-Fm zwischen die Rotenberg-Fm einschiebt bzw. mehr oder weniger synchron, aber einer anderen $\mathrm{Fa}$ zies (geringere Wassertiefe) zugehörig ist. Hingegen dürften die üblicherweise ebenfalls zur Rotenberg-Fm gerechneten Anteile mit grünlichen Radiolariten deutlich jünger und in die Unterkreide zu stellen sein - sie werden bei Andrzej Ślączka et al. demnach als jüngerer Anteil der FasslgrabenFm geführt. ${ }^{8}$

Die Gegenüberstellung der geologischen Karte (Abb. 1) ${ }^{9}$ und der Kartierung von mittlerweile über 20 gesicherten Fundstellen mit Bergbauzusammenhang (Abb.2; Stand Ende 2018) zeigt ein völlig deckungsgleiches Bild letzterer

\footnotetext{
5 Trauth 1930.

6 Schnabel 1998.

7 Pfersmann 2013. - ŚlączKa et al. 2018.

8 ŚLĄCZKa et al. 2018.

9 Schnabel 1998.
} 


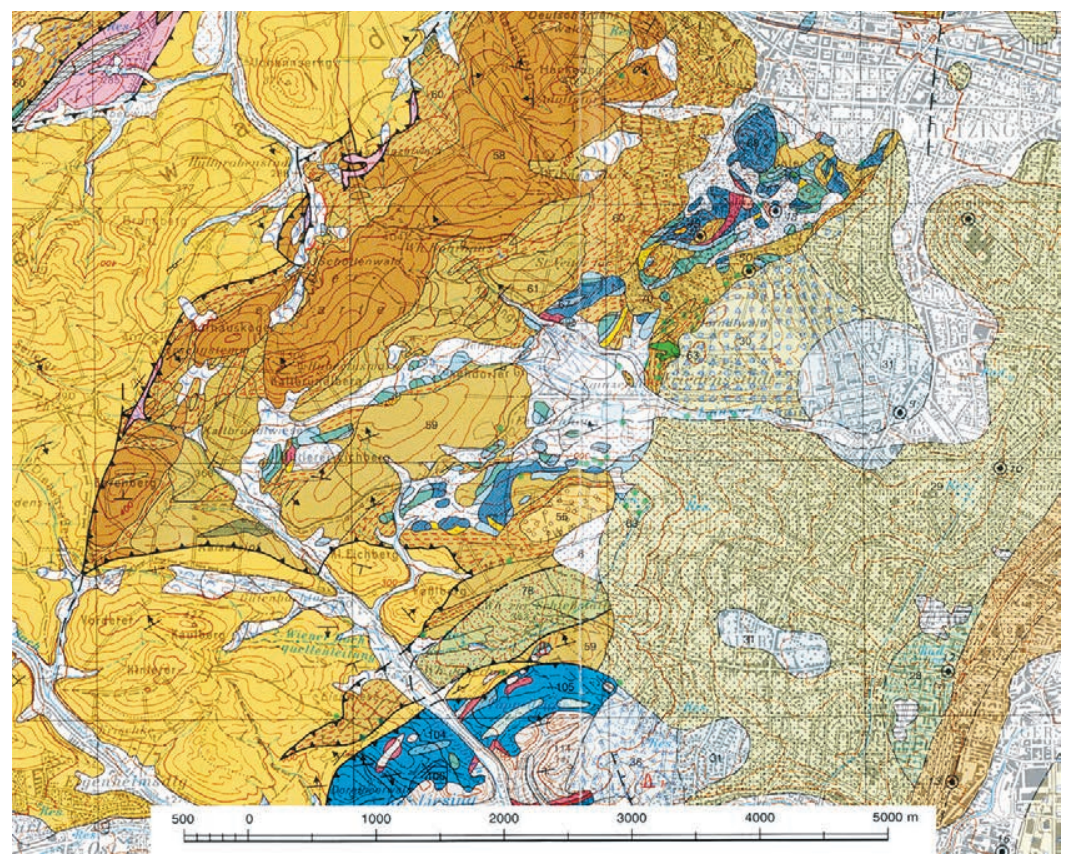

Abb. 1. Geologische Karte (@) GBA). - Türkis: Rotenberg-Fm. - Hellblau: Fasslgraben-Fm.

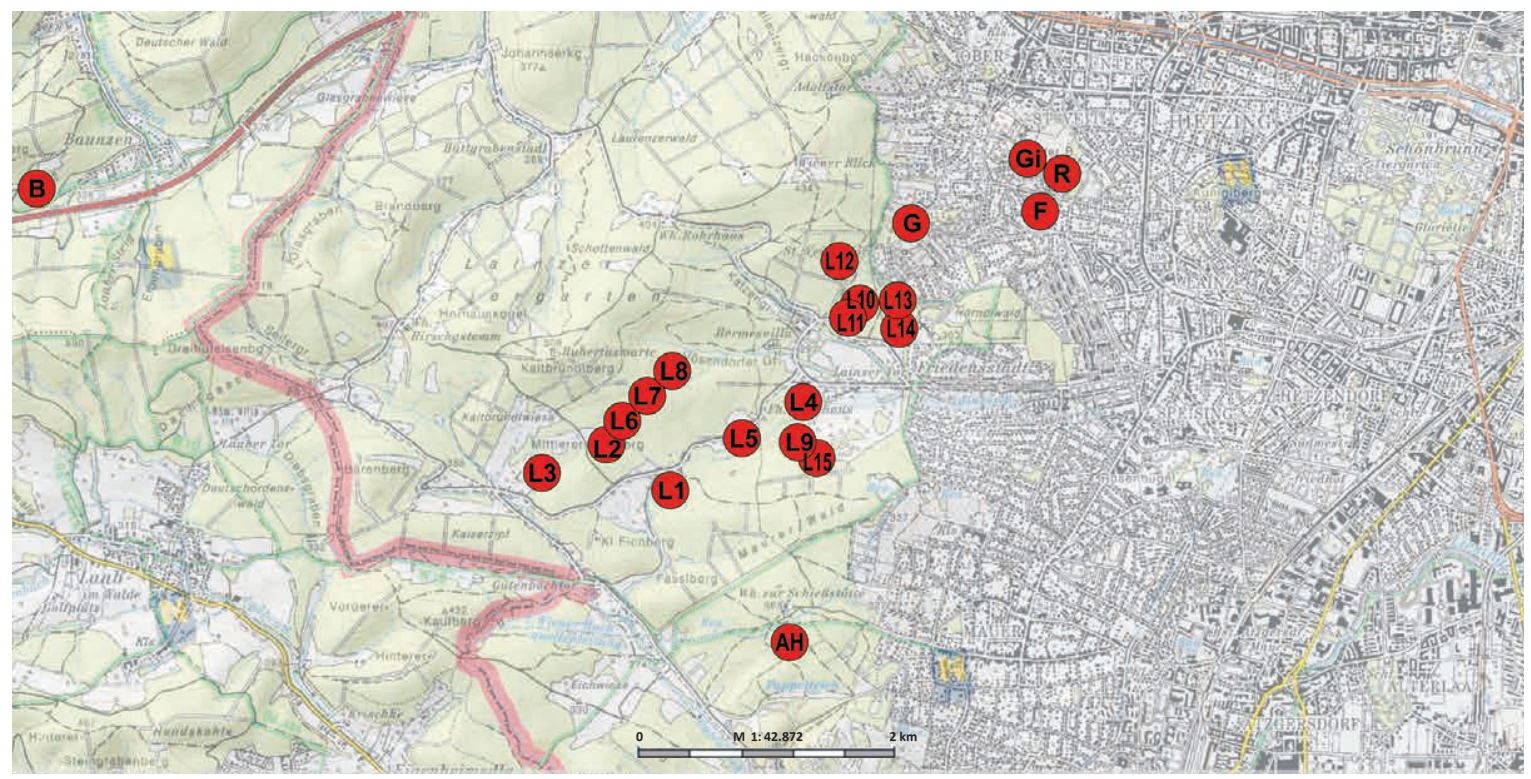

Abb. 2. Gesicherte Fundstellen mit Bergbauzusammenhang. - B: Baunzen (Purkersdorf/NÖ). - R: Roter Berg. - Gi: Girzenberg. - F: Flohberg. - G: Gemeindeberg. - AH: Antonshöhe. - L: Lainzer Tiergarten 1-15 (Kartengrundlage: NÖ-Atlas 4.0, ( ) Land Niederösterreich; Grafik: O. Schmitsberger).

mit der Rotenberg-Fm und der sie regelhaft begleitenden Fasslgraben-Fm, inklusive der „Ausreißer“ im Nordwesten (Baunzen bei Purkersdorf) ${ }^{10}$ und Südosten (Antonshöhe). ${ }^{11}$ Etwa die Hälfte der relevanten Fläche der zentralen SVK liegt aber im heute dicht verbauten Stadtgebiet - mit einer hohen Anzahl unerkannt bzw. unbeachtet zerstörter oder überbauter Bergbaubefunde muss daher gerechnet werden.

10 Brandl, Trnka 2014. - Brandl, Schmitsberger, Trnka 2018. 11 Ruttkay 1970. - Trnka 2014.
Die Fundstelle in der Baunzen liegt zwar in einem Zug der Grestener- bzw. Hauptklippenzone, Siegmund Prey schreibt aber definitiv von „Klippen vom Typus St. Veit“. ${ }^{12}$ Aufgrund dessen und wegen der noch nicht völlig klaren geologisch-tektonischen Zuordnung der Antonshöhe ${ }^{13}$ verwenden wir als Sammelbegriff für das Rohmaterial daher die Bezeichnung „Wiener Klippenzonen-Radiolarit“.

12 PREY 1979.

13 Z. B. Prey 1991. 


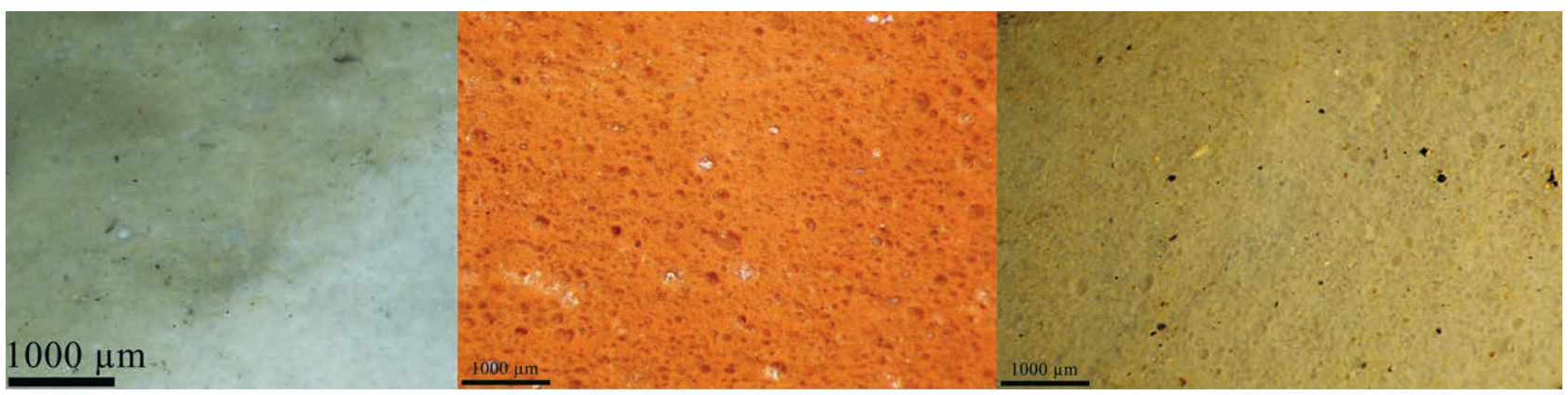

Abb. 3. Radiolarite der St. Veiter Klippenzone. - Links: Typ Gemeindeberg. - Mitte: Typ Antonshöhe. - Rechts: Typ Baunzen (Mikrofotos: M. Brandl).

\section{Das Rohmaterial Radiolarit}

Das Hauptinteresse der prähistorischen Bergleute in unserer Forschungsregion lag an den meist roten oder grünen Radiolariten der Rotenberg-Fm, untergeordnet wurden aber auch braungraue bis schwarzblaue Radiolarite der Fasslgraben-Fm genutzt. Bei den Radiolariten der Rotenberg-Fm unterscheiden wir vorläufig drei unterschiedliche Typen: den Typ Gemeindeberg, den Typ Antonshöhe und den Typ Baunzen (Abb. 3). ${ }^{14}$

Die Definition und Abgrenzung gegenüber anderen Lagerstätten bzw. Varietäten erfolgt mittels Multi Layered Chert Sourcing Approach (MLA) in drei Schritten: erstens makroskopisch, zweitens mikroskopisch und drittens geochemisch. ${ }^{15}$ Diese Methode beruht auf der Kombination makroskopischer Gruppenbildung und anschließender stereomikroskopischer Feinzuordnung. Aus den daraus resultierenden mikroskopischen Gruppen werden ausgewählte Stücke geochemisch auf Spurenelementgehalte untersucht. Für die Spurenelementanalyse wird Laser Ablation Inductively Coupled Mass Spectrometry (LA-ICP-MS) eingesetzt. Die geochemischen Daten werden statistisch mittels Compositional Data Analysis (CODA) ausgewertet, um eine optimale Gruppenzuweisung bzw. Differenzierung zu erzielen.

Problematisch dabei ist, dass Radiolarite mikroskopisch schwierig zu differenzieren sind. ${ }^{16}$ Bei besonders charakteristischen Ausprägungen (viele Negative von Calcitkristallen etc.) können zwar SVK-Radiolarite mit hoher Wahrscheinlichkeit des Zutreffens der Bestimmung auch mikroskopisch identifiziert werden, meist ist zur endgültigen Abklärung aber eine geochemische Analyse

14 Brandi, Trnka 2014. - Brandl et al. 2014.

15 BRANDL 2016.

16 Vgl. z. B. Ruiz-Ortiz, Bustillo, Molia 1989.

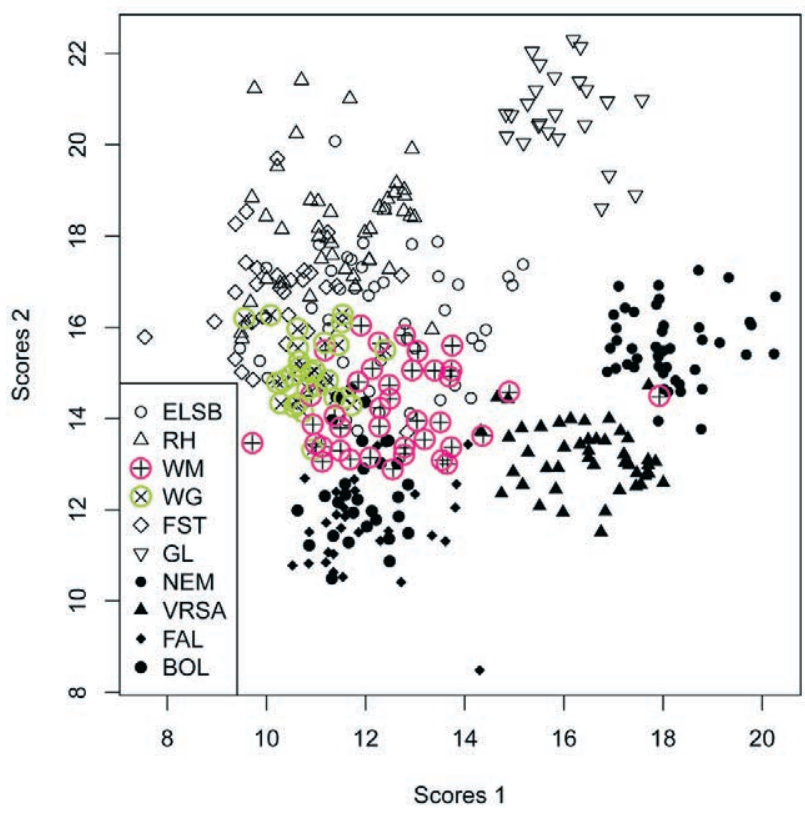

Abb. 4. Grafische Darstellung der Auswertung von Spurenelementanalysen unterschiedlicher Radiolaritressourcen (Fischer resolution plot). - Volle Symbole: Radiolarite der Pienidischen Klippenzone. - Leere Symbole: Radiolarite der Nördlichen Kalkalpen. - WM: Wien-Mauer. - WG: Wien-Gemeindeberg (nach BRANDL et al. 2014, Grafik: P. Filzmoser, Bearbeitung: O. Schmitsberger).

nötig, was zeitaufwändig und damit leider kostenintensiv ist. Diese Charakterisierung ist jedoch die Voraussetzung dafür, die Klippenzonen-Radiolarite in archäologischen Fundkomplexen einem bestimmten Vorkommen zuweisen zu können. Nur so wird zukünftig eine gesicherte Aussage zu den steinzeitlichen „Wiener Exporten“ und deren Reichweite möglich sein. Eine vereinzelt in der Literatur angeführte Verwendung von „Wiener Klippenzonen-Radiolarit" im Neolithikum bis beispielsweise nach Bayern ${ }^{17}$

17 Grillo 1997. 

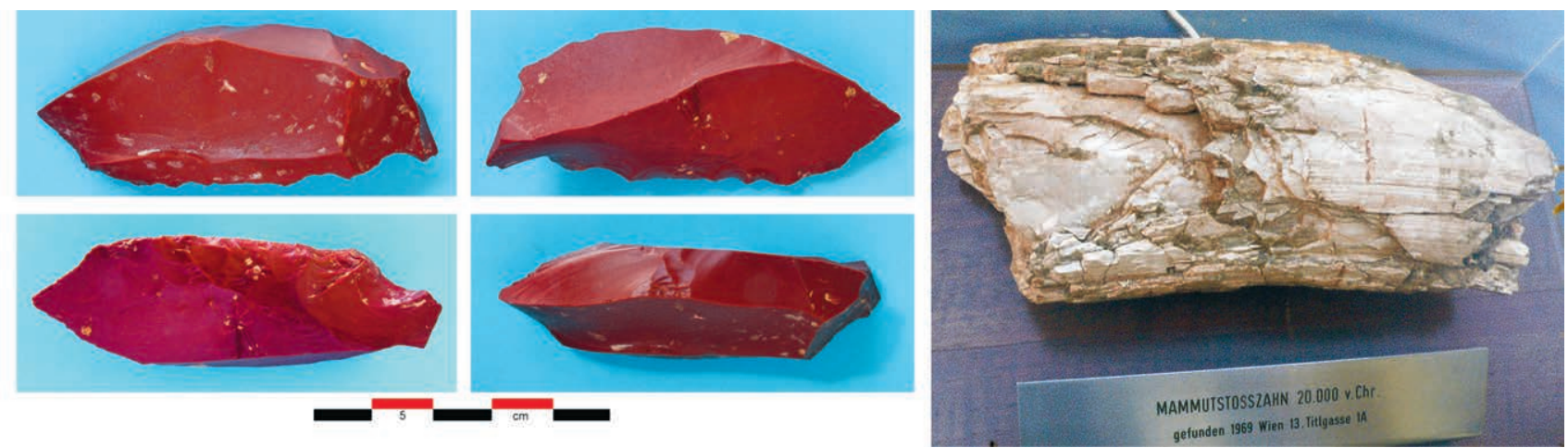

Abb. 5. Paläolithische Funde aus Wien 13, Titlgasse 1A. - Schaberartig retuschiertes Artefakt aus Radiolarit (Bezirksmuseum Hietzing, Foto: G. Trnka). - Beim Grundbesitzer befindliches Mammut-Stoßzahnfragment (Foto: Forschungsgruppe Quartärarchäologie, OREA/ÖAW).

ist aus den angeführten Gründen sehr zu hinterfragen, wenn auch nicht völlig unwahrscheinlich.

Nach ersten geochemischen Untersuchungen und deren Auswertung (z. B. Plot Barium gegen Magnesium $)^{18}$ liegen die SVK-Radiolarite mit den Proben vom Gemeindeberg und der Antonshöhe summarisch zwischen den kalkalpinen Radiolariten und jenen der Pienidischen Klippenzone der Karpaten, allerdings mit größerer Affinität zu den nördlichen Kalkalpen (Abb. 4). Neuere, noch unpublizierte Ergebnisse lassen aber die Möglichkeit einer weit detaillierteren Unterscheidung erwarten.

\section{Prähistorische Nutzung von „Wiener Klippenzonen-Radio- larit“"}

Radiolarit war - als Mitglied der Hornsteingruppe ${ }^{19}$ - während der gesamten Steinzeiten und darüber hinaus ein wichtiger Rohstoff für alle Arten von Geräten, die eine scharfe, schneidende Arbeitskante benötigen. Die Nutzung des Wiener Materials erfolgte schon im Paläolithikum, wie etwa in Wien 13, Titlgasse, ${ }^{20}$ und zwar anscheinend nicht nur im heutigen Wiener Gebiet, sondern auch im Raum Krems in Niederösterreich.

Ein eigentlicher Bergbau auf Radiolarit fand dann ab dem bzw. im Neolithikum statt, Belege für die Nutzung des hiesigen Materials reichen dabei bis ins späte Endneolithikum (Kosihy-Čaka/Makó-Gruppe) und sogar bis in die Bronzezeit. Die Verwendung von „Wiener

18 Brandl et al. 2014, Fig. 7.

19 Brandl 2013-2014.

20 Felgenhauer, Mossler 1969. - Schmitsberger, Neugebauer-Maresch 2018b. - Schmitsberger, Neugebauer-Maresch 2018c.

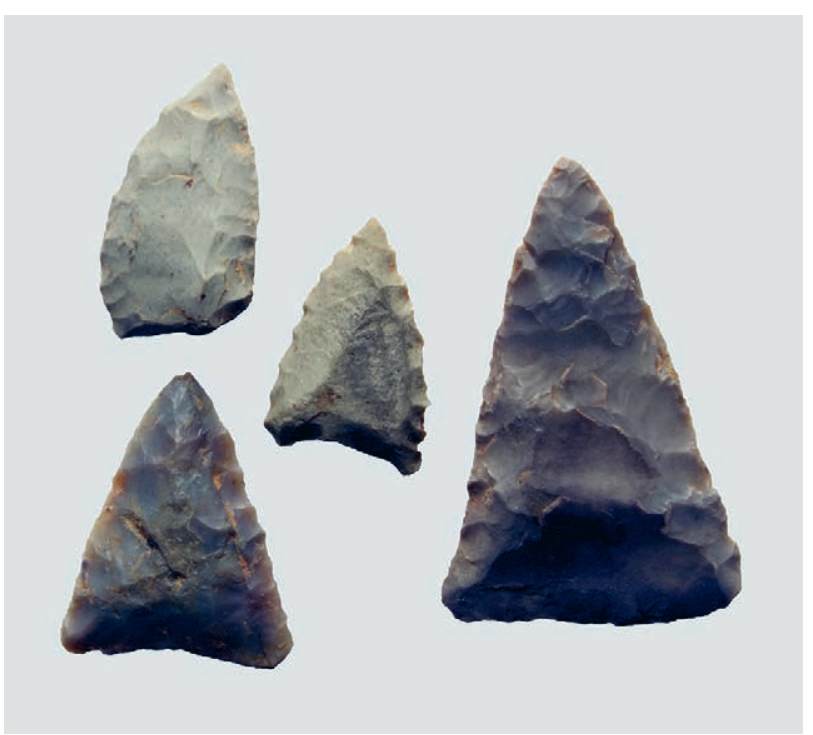

Abb 6. Spätneolithische Pfeilspitzen aus „Wiener KlippenzonenRadiolarit" von der Siedlung am Gemeindeberg (Sammlung Wienmuseum, Foto: M. Penz, Bearbeitung: O. Schmitsberger).

Klippenzonen-Radiolarit“ ist also für einen Zeitraum von mindestens 20.000 Jahren belegt (Abb. 5 und 6). ${ }^{21}$

\section{Neolithischer Bergbau in und um Wien}

\subsection{Bereits bekannte Abbaustellen}

Altbekannte Fundstellen mit eindeutigen Bergbauspuren sind in Wien lediglich die Antonshöhe und der Flohberg.

Auf der Antonshöhe bei Mauer wurde Anfang des 20. Jhs. ein regulärer Bergbau (Bergwerk mit Schächten) durch einen Steinbruch angeschnitten (Grabungen Bayer

21 Schmitsberger, Penz 2017, 158-160. 


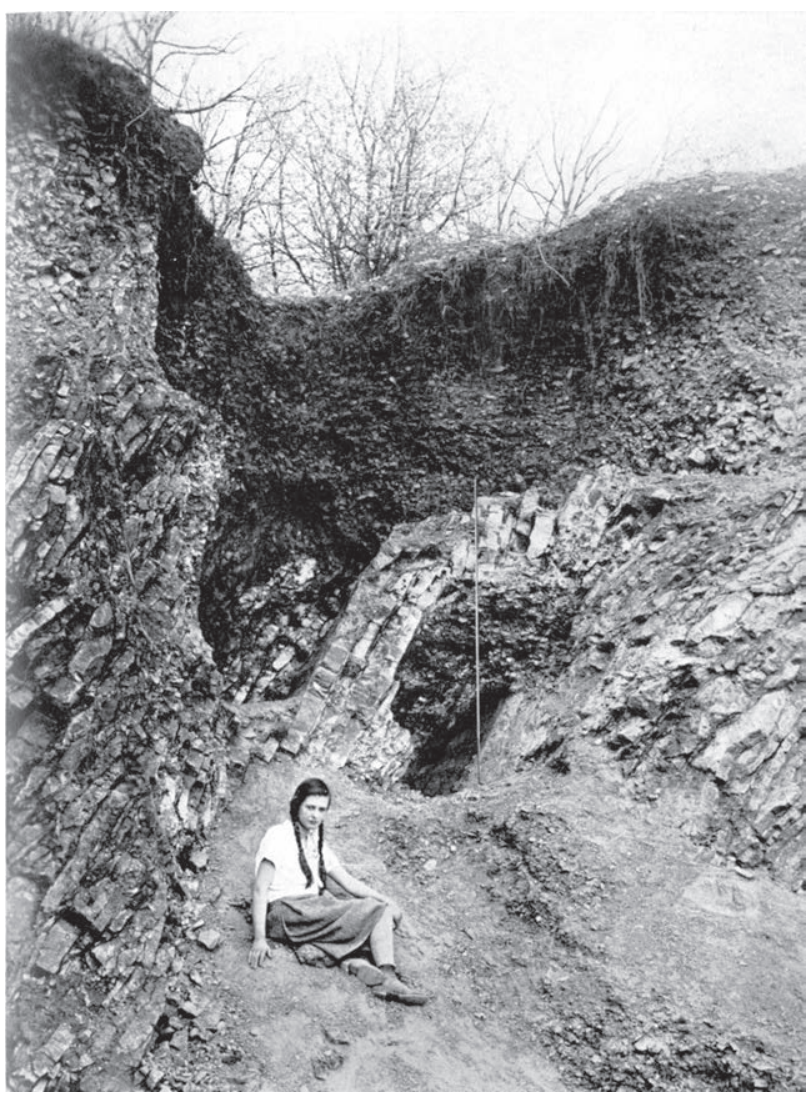

Abb. 7. Bergbau Antonshöhe, Grabung Bayer (Foto: K. Kriegler).

1929/30; Abb. 7) und zu großen Teilen zerstört. Der Bergbau datiert ins letzte Drittel des 5. Jts. v. Chr., also an den Übergang vom Mittelneolithikum zur Kupferzeit. ${ }^{22}$ Außer vielen Schlag- und Klopfsteinen aus unterschiedlichen Materialien, zum Teil aus Äxten und Beilen in Sekundärverwendung, wurde eine größere Anzahl an Geweihgezähen beobachtet und zum Teil auch geborgen. ${ }^{23}$

Der Abbau am Flohberg ${ }^{24}$ ist wenig bekannt bzw. wurde bislang kaum beachtet. Nach spärlichen Bei- und Umgebungsfunden ist er wohl mittelneolithisch, der Beginn könnte jedoch eventuell schon im Altneolithikum gelegen haben. ${ }^{25}$ Nach dem dokumentierten Profil dürfte es sich um einen Pingen- bzw. Kuhlenbau gehandelt haben (Abb. 8), aufgrund des „Zufallsprofils“ in der Baugrubenwand bleibt aber die tatsächliche Tiefe der Abbaugrube unbekannt. An Bergbaugeräten sind zwei Schlägel aus Quarz(it)geröllen überliefert (Abb. 9), spärliche Reste rot-weiß bemalter

22 TRNKa 2014.

23 RutTKAy 1970

24 Dollinger, Wenty 1962.

25 Schmitsberger, Penz im Druck a.
PROFIL DES BAUAUFSCHLUSSES

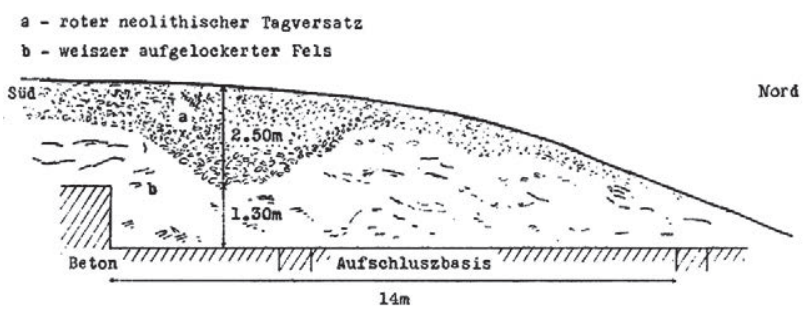

Abb. 8. Bergbau Flohberg, Profilskizze (nach Dollinger, Wenty 1962).

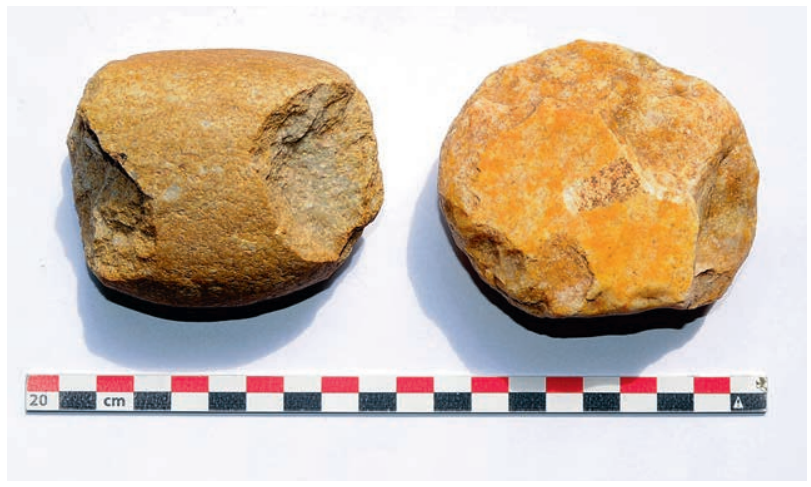

Abb. 9. Bergbau Flohberg, Schlägel bzw. Abbaugeräte aus Quarzit (Sammlung Dollinger im Amtshaus Hietzing, Foto: M. Penz).

Keramik datieren zumindest diesen einen Befund in die Stufe MOG IIa der Lengyelkultur, also in die Mitte des 5. Jts. v. Chr. Nach den alten Fundberichten zu schließen, erstreckte sich anschließend an die Abbaustelle vermutlich eine zugehörige Siedlung. Der Flohberg gehört wie der Girzenberg zum Komplex „Roter Berg“ - für diese beiden anderen Klippen sind prähistorische Abbauaktivitäten durch entsprechende Oberflächenfunde belegt. ${ }^{26}$

\subsection{Aktuelle Geländetätigkeit}

\subsubsection{Prospektion}

Im Zuge der Geländeprospektion wurde seit 2016 eine Vielzahl neuer Fundstellen entdeckt, vor allem im von neuzeitlicher Verbauung verschont gebliebenen Lainzer Tiergarten - allein dort sind bis dato 15 Abbau- bzw. Nutzungsstellen zu verzeichnen (Abb. 10). Diese hier alle im Detail anzuführen und zu beschreiben, würde den vorgegebenen Rahmen

26 Schmitsberger, Penz im Druck a. - Schmitsberger, Penz im Druck c. 


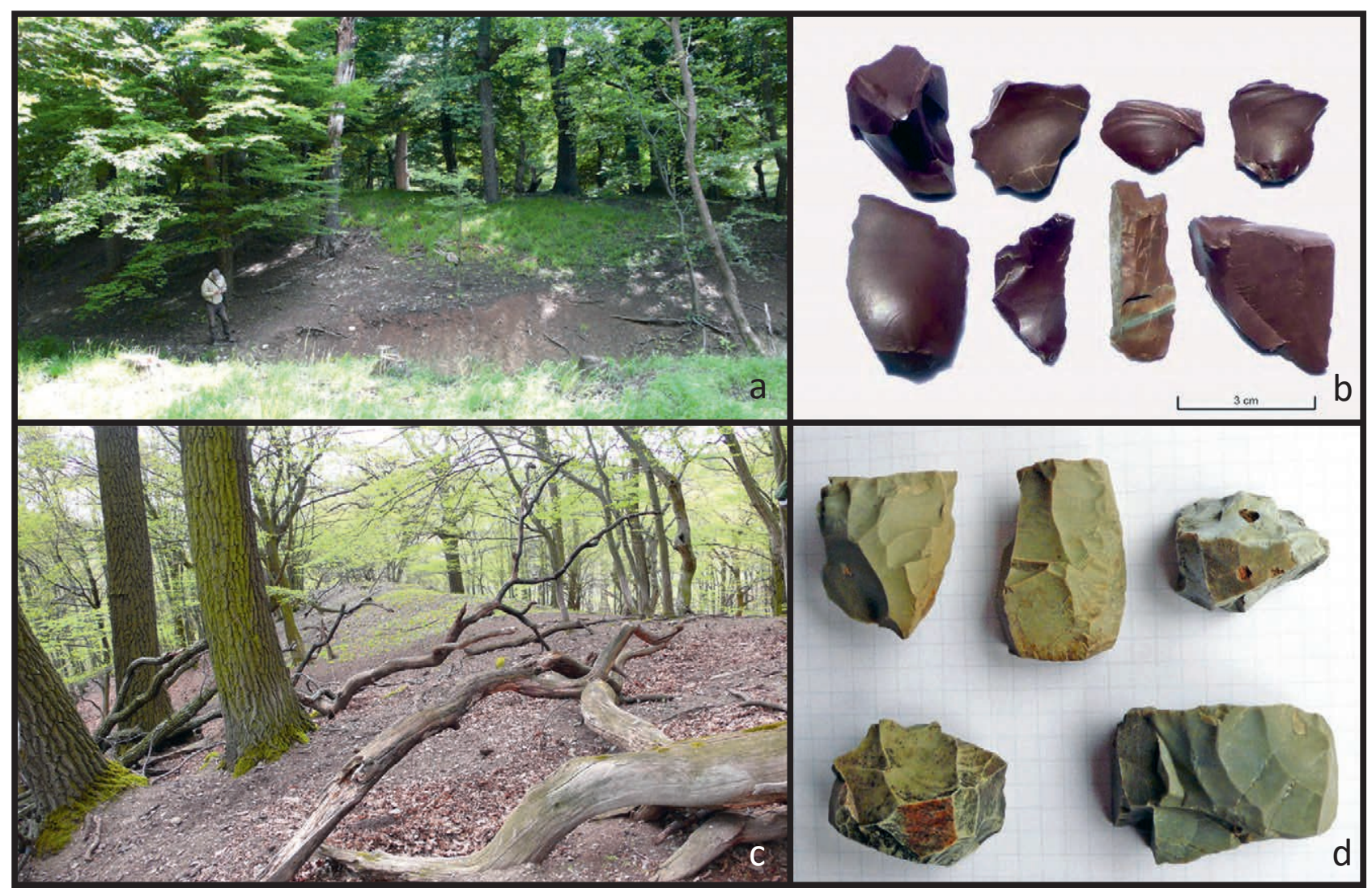

Abb. 10. Lainzer Tiergarten. - a. Fundstelle 1, Halde. - b. Fundstelle 1, Abschläge. - c. Fundstelle 3. - d. Fundstelle 3, Nuclei (Fotos: O. Schmitsberger, M. Penz).

bei weitem sprengen; es sei hiermit also nur auf die entsprechenden Vorberichte verwiesen. ${ }^{27}$

Diese Fundstellen wurden durch bergbautypische Oberflächenfunde wie artifizielle Trümmerstücke (Abbauschutt), Entrindungsabschläge, precores, Schlagsteine etc. identifiziert, die Nutzung bzw. der Abbau sowohl von Radiolarit-Knollen als auch von gebanktem Material ist nachgewiesen.

Die Tätigkeit im Naturschutzgebiet Lainzer Tiergarten beschränkte sich nur auf oberflächige Prospektion in Form von Geländebegehungen, bislang wurden keine Grabungen oder sonstigen Bodeneingriffe durchgeführt, sondern lediglich eine reine Fundstellenkartierung. Das Gebiet des Tiergartens weist zwar keine Verbauung auf, aber hier ist

27 Penz 2016. - Penz, Schmitsberger 2016. - Schmitsberger, Penz 2017a. - Schmitsberger, Penz 2017b. - Schmitsberger, Penz 2018a. - Schmitsberger, Penz 2018b. - Schmitsberger 2019a. Schmitsberger, Neugebauer-Maresch 2019. - Schmitsberger im Druck a. - Schmitsberger im Druck b. - Schmitsberger, Penz im Druck b. ein Problem anderer Art hinderlich, nämlich eine Vielzahl ehemaliger kleiner Steinbrüche, die bevorzugt an Klippenaufragungen angelegt wurden. Besser ist die Situation diesbezüglich bei im Gelände unauffälligen Klippen, also solchen, die sich nicht morphologisch, sondern nur durch die vorkommenden Gesteine als solche zu erkennen geben; Friedrich Trauth spricht von „Krypto-Klippen“ im Gegensatz zu „morphologischen Klippen“. ${ }^{28}$ Allerdings ist hier wiederum durch eine stärkere Humusauflage, dadurch bedingt mehr Bewuchs, und oft eine Laubbedeckung infolge nur schwacher Hangneigung und geringer Erosionsrate eine schlechte Sichtbarkeit gegeben, was das Auffinden solcher Fundstellen erschwert.

Als Kriterien für eine Interpretation der bei den Begehungen festgestellten Fundstellen als „Bergbauspuren“ diente dabei das Zutreffen der folgenden drei Punkte: a) Artefakte direkt am Rohmaterial-Vorkommen, b) beinahe nur Schlagabfälle bzw. „Abbauschutt“ und kaum oder keine

28 Trauth 1930, 107. 
modifizierten Geräte wie in Siedlungen (Kratzer, Messer, Pfeilspitzen etc.), c) kaum oder keine typischen Siedlungsfunde wie Keramik, Reibplatten etc.

Eine Ausnahme bildete dabei die Fundstelle 12, von welcher neben Radiolaritabfällen auch sehr wenig Keramik (feinchronologisch noch nicht zuordenbare kleine Fragmente) und ein Reibplattenbruchstück vorliegen. Eventuell handelt es sich hier um eine „Außenstelle“ oder „temporäre Spezialsiedlung" direkt am Radiolaritvorkommen.

Offensichtlich liegen an einzelnen Stellen der SVK sehr unterschiedliche Nutzungsintensitäten vor bzw. haben wir es mit Fundstellen unterschiedlicher "Qualität“ zu tun: die Hinterlassenschaften der Radiolarit-Abbau- bzw. Gewinnungsaktivitäten reichen von nur wenigen Abschlägen direkt am Rohmaterialvorkommen als spärlicher Beleg der Nutzung desselben (z. B. Lainzer Tiergarten 9 und 12) bis hin zu regelrechten Schlagabfallhalden (z. B. Lainzer Tiergarten 1, 15 und Baunzen). Nach derzeitigem Wissensstand wurden manche der Stellen also anscheinend nur minimal bzw. sporadisch genutzt, andere wiederum sehr intensiv und vermutlich über einen längeren Zeitraum hinweg.

Nach den wenigen, typotechnologisch beurteilbaren Artefakten wie z. B. bestimmten Kerntypen dürften die meisten der oberflächig auffindbaren, durch Schlagabfälle gekennzeichneten Aktivitätsbereiche dem Mittel- bis Spätneolithikum zuzurechnen sein. Mit einem deutlich früheren Einsetzen intensiver bzw. systematischer Nutzung der Rohmaterialressourcen ist jedoch zu rechnen, wie Artefakte aus entsprechendem Material, beispielsweise aus linearbandkeramischen Siedlungen zeigen.

Eher sporadisch prospektiert wurde jedoch auch im angrenzenden Niederösterreich, z. B. die Abbaustelle Purkersdorf-Baunzen (Abb. 11). Hier erfolgte die Nutzung nach typologisch-technologischen Merkmalen der Artefakte anscheinend vor allem im Spätneolithikum. ${ }^{29}$

Die Silizitvorkommen der Flyschzone wurden bisher nur randlich mitbehandelt, aber auch dort fanden sich eindeutige Hinweise auf eine Nutzung bzw. einen Abbau des vorkommenden Rohmaterials „Flyschsilizit“, Spiculit und eingekieselter feinkörniger Glaukonit-Quarzsandsein, so etwa im 14. und 19. Wiener Gemeindebezirk. ${ }^{30}$

\subsubsection{Grabung}

Der Gemeindeberg im 13. Bezirk ist eine der am längsten bekannten prähistorischen Fundstellen in Wien. Auf der

29 Brandl, Schmitsberger, Trnka 2018.

30 SieheSchmitsberger, Neugebauer-Maresch 2018b.-SchmitsBerger, Neugebauer-Maresch 2019.
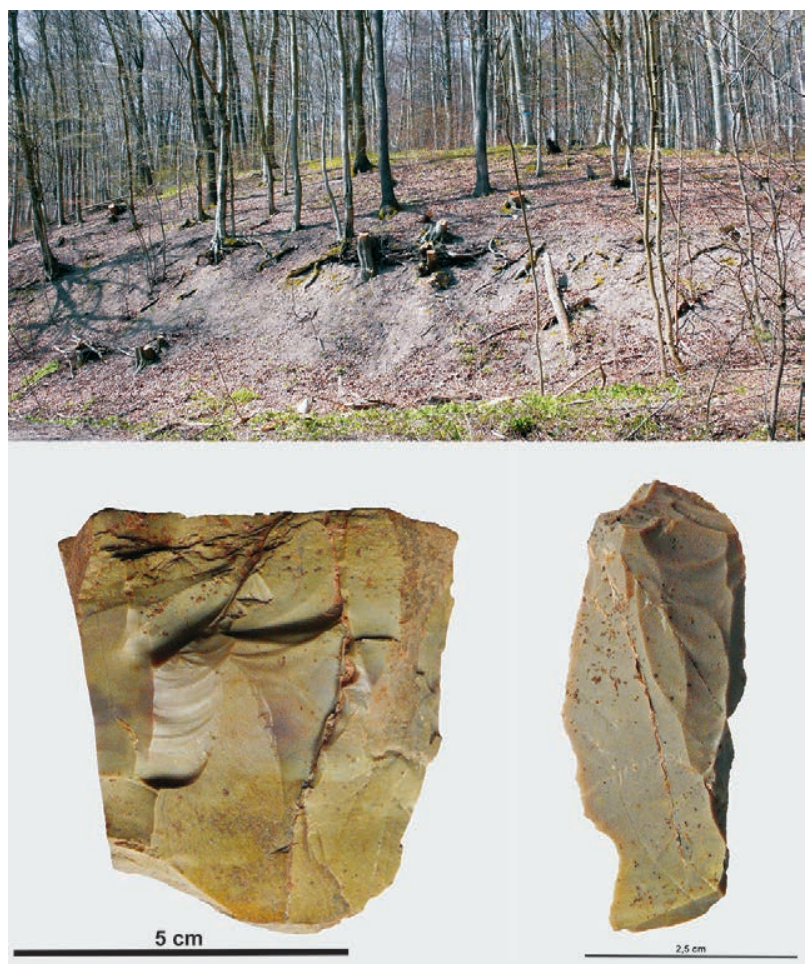

Abb. 11. Baunzen bei Purkersdorf, Radiolarithalde. - Nucleus und klingenförmiger Abschlag (Fotos: O. Schmitsberger).

Kuppe des exponierten Hügels existierten zwei Siedlungen aus unterschiedlichen Phasen des Spätneolithikums bzw. der Kupferzeit (Frühe Badener Kultur/Bolerázgruppe, 4. Jt. und Jevišovice-Kultur, 3. Jt. v. Chr.), dort fanden Altgrabungen unterschiedlicher Wissenschafter vom 19. Jh. bis in die 1960er Jahre statt. ${ }^{31}$ Westlich anschließend befindet sich am Nordhang ein ausgedehntes Abbau- und Haldengebiet $^{32}$ - ursprünglich wohl auch am Südhang, dieser ist aber heute weitgehend verbaut. Trotz der langen Forschungstradition wurde der Bergbau-Aspekt erstaunlicherweise bisher kaum beachtet oder auch nicht bzw. nur unzureichend erkannt. Da hier Siedlungsstelle und Abbaugebiet unmittelbar nebeneinander liegen, ist für die kupferzeitlichen Siedlungsbefunde definitiv eine Interpretation als Bergbausiedlungen anzunehmen. Unter den aus den Altgrabungen von Josef Bayer, Jaroslav Czech von Czechenherz und Josef Fritz Kastner stammenden Funden von der Siedlungsfläche am Gemeindeberg im Depot des Wien Museums ist der für Siedlungsfunde weit überdurchschnittlich hohe Anteil an Geweihgeräten bzw. -gezähen und Schlagsteinen besonders auffällig - dies stützt diese Interpretation zusätzlich.

31 Penz 2007.

32 Schmitsberger 2019b. 


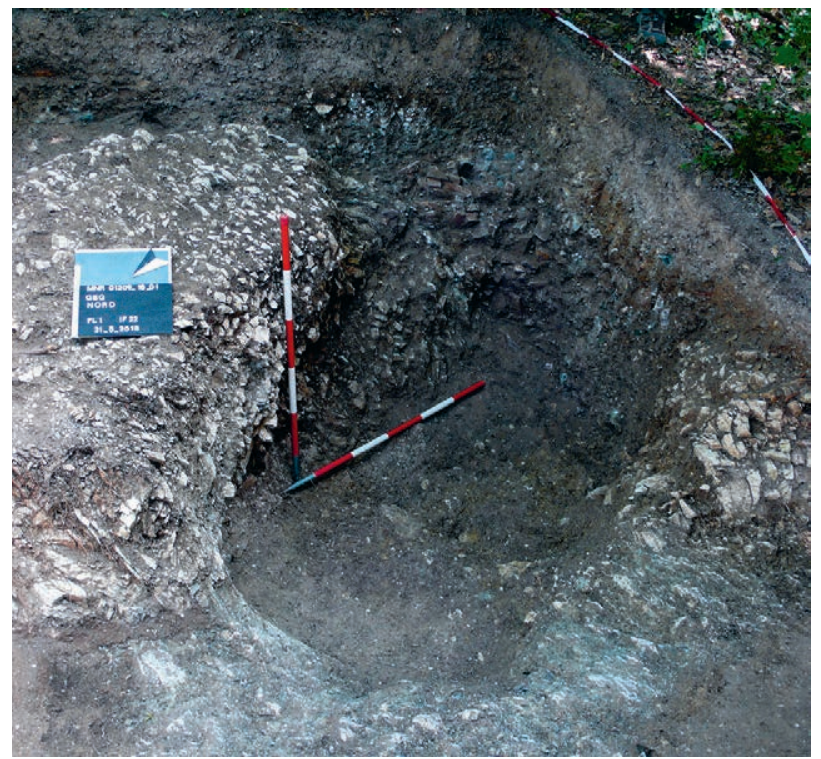

Abb. 12. Grabung Gemeindeberg 2018, Abbaugrube (Schacht?) Objekt 2, Endzustand, Sohle nicht erreicht (Foto: O. Schmitsberger).

2017 waren außerhalb der Siedlungsfläche nach einer Wegverbreiterung in der Böschung eingetiefte Befunde entdeckt worden. ${ }^{33}$ Daraufhin wurde 2018 in einer kurzen Kampagne das „Forststraßen-Böschungsprofil“ dokumentiert und zudem eine kleine Flächengrabung durchgeführt. Dabei gelang der Nachweis von Obertagebau mit Feuersetzen und daran anschließend ein in die Tiefe gehender Abbau. Die Sohle der durch die Grabung untersuchten Grube (Objekt 2) wurde noch nicht erreicht (Abb. 12), es ist daher noch unklar, ob es sich „nur“ um eine tiefere Abbaugrube handelt oder ob ein echter Schacht vorliegt. ${ }^{34}$ Nach ersten ${ }^{14} \mathrm{C}$-Datierungen ist Objekt 2 mittelneolithisch - dies ist weniger überraschend als man annehmen möchte, da im Mittelneolithikum in der SVK anscheinend ein regelrechter „Bergbau-Boom“ herrschte, wie die Abbaustellen Roter Berg, Flohberg, Antonshöhe (und wohl einige weitere) zeigen.

Dass insgesamt erst drei Bergbaue bzw. Abbaustellen auch durch entsprechende Befunde nachgewiesen sind, mag wenig erscheinen, ist jedoch sehr zu relativieren: Denn in allen Bereichen anstehender Rotenberg-Fm mit qualitätsvollem Radiolarit, wo bei tiefer reichenden Bodeneingriffen näher nachgesehen wurde, kamen auch entsprechende Befunde zutage. Es ist demnach wohl gerechtfertigt, vergleichbare Befunde auch für viele (oder die meisten, wenn nicht

33 SChMitsberger 2019b.

34 Schmitsberger im Druck c. - Brande, Penz, Schmitsberger im Druck. sogar alle) jener Stellen, von welchen bislang lediglich bergbautypische Oberflächenfunde vorliegen, anzunehmen.

\section{Zugehörige Siedlungen - Archivarbeit und Altbestände}

Siedlungen, sozusagen die umgebende Bergbau-Infrastruktur, sind natürlich mit einzubeziehen, und zwar in diachroner Betrachtungsweise für jeweils einzelne Zeitabschnitte. Die neolithischen Siedlungen liegen aber mit Ausnahme einiger Höhensiedlungen überwiegend im heute dicht verbauten Stadtgebiet, was diesbezügliche Forschung besonders erschwert. Es sind daher fast nur punktuelle Informationen anhand meist schlecht oder gar nicht befundeter bzw. dokumentierter Altfunde vorhanden. Ein Anfang wurde hier bereits mit der Zusammenstellung „bergbaurelevanter Siedlungen“ im Gebiet des Lainzer Tiergartens ${ }^{35}$ gemacht, und mit der mit aufwändiger Archivrecherche verbundenen Darstellung der frühneolithischen, auf den Bergbau in der SVK Bezug nehmenden Siedlungsstellen im 13. Wiener Gemeindebezirk. Auch hier zeigte sich, überwiegend anhand unpublizierter Archivalien und Sammlungs-Altbestände, dass mit dem Einsetzen eines regulären Bergbaus auf Radiolarit bereits während der Linearbandkeramik zu rechnen ist. $^{36}$

Für das Spätneolithikum ist festzuhalten, dass Siedlungsstellen sowohl im Lainzer Tiergarten in der Nähe der Radiolaritvorkommen - jedoch nicht direkt auf diesen - bekannt sind (Fasslberg, Nikolaiberg), überwiegend liegen sie aber außerhalb, vor allem im Wiental (Satzberg, Georgenberg bei Purkersdorf und andere).

\section{Fazit}

Die Dimensionen der neolithischen Radiolaritgewinnung in der SVK wurden bisher offenbar völlig unterschätzt. Es handelt sich unseren Erkenntnissen zufolge keineswegs um vereinzelte Befunde, wie bislang angenommen wurde, sondern es kann schon jetzt, obwohl unsere diesbezüglichen Forschungen erst am Anfang stehen, gezeigt werden, dass hier ein prähistorisches Bergbaugebiet von europäischem Rang, vergleichbar mit Krumlovsky Les ${ }^{37}$ in Mähren oder dem Bakony-Gebirge/Szentgál in Westungarn, ${ }^{38}$ vorliegt.

Die Anfänge des Wiener Radiolarit-Bergbaus dürften spätestens im Altneolithikum (Linearbandkeramik) gelegen haben, ${ }^{39}$ eine Blütezeit scheint er im Mittelneolithikum

\footnotetext{
35 Schmitsberger, Penz 2018a, 123-124.

36 Schmitsberger, Penz im Druck a.

37 Oliva 2010.

38 Biró et al. 1983-1995.

39 Hinweise auf ein eventuell noch früheres Einsetzen desselben sind vorhanden, müssen aber erst verifiziert werden.
} 
(Lengyelkultur) erlebt zu haben, eine offenbar intensive spätneolithische „Nachnutzung“ ist ebenfalls sicher belegt, einerseits durch das häufige Vorhandensein entsprechenden Rohmaterials in den Siedlungen, andererseits unter anderem durch ein charakteristisches Keramikfragment vom Schlagplatz „Sterndlwiese“ in der unmittelbaren Nachbarschaft der Antonshöhe. ${ }^{40}$

Die Ausdehnung des Bergbaugebiets reicht vom Gütenbachtal im Südwesten bis zum Komplex Roter Berg/ Girzenberg/Trazerberg im Nordosten und verfügt über seitliche „Ausreißer“ wie Antonshöhe und Baunzen. Die Bergbaurelikte streuen somit über eine Längserstreckung von mehr als fünf Kilometern und eine maximale Breite im Lainzer Tiergarten (nördlicher und südlicher Klippenzug) ${ }^{41}$ von etwa einem bis eineinhalb Kilometern (ohne die „Ausreißer“). Wir sprechen hier also von einem (Kern-)Gebiet von etwa $5,5 \mathrm{~km}^{2}$, in welchem der prähistorische Bergbau umging - und dies bezeichnet wohlgemerkt nur die eigentlichen Abbaustellen ohne die umgebende „Infrastruktur“ wie Support-, Produktions-, Distributionssiedlungen etc.

Dabei sind jedoch die eigentlichen Abbaustellen nicht isoliert $\mathrm{zu}$ sehen, sondern inklusive umgebender regulärer Siedlungen, (temporärer) Spezialsiedlungen und "Sondernutzungsstellen“. ${ }^{42}$ Sie sind also in eine infrastrukturelle Bergbauumgebung eingebunden, welche einerseits zur Versorgung des Bergbaus dient, sowie andererseits zur Verarbeitung des abgebauten Rohmaterials, also für Produktion und Distribution. Erst wenn diese Nutzung der umgebenden Landschaft in die Betrachtung mit einbezogen wird, ist auch die sozioökonomische Dimension des Bergbauphänomens zu erfassen. Dieses enorme Forschungsund Erkenntnispotential sollte unbedingt ausgenutzt werden.

\section{Danksagung}

Wir danken Ch. Blesl (BDA), G. Trnka und M. A. Götzinger (Universität Wien), F. D. Eschner (Stadt Wien, MA 22), A. Mrkvicka und L. Fidler (Stadt Wien, MA 49), sowie für ihre Fundmeldungen R. Kunz, D. Kowarik und B. Dekan.

40 Felgenhauer 1967. - Penz, Reiter 2012, 170.

41 Siehe Schmitsberger, Penz 2018a, Abb. 2.

42 Z. B. Hörndlwald siehe Schmitsberger 2015. - Schmitsberger, Penz im Druck a.

\section{Literatur}

ANDREFSKY 2009

W. ANDREFSKY, The analysis of stone tool procurement, production and maintenance, Journal for Archaeological Research 17, 2009, 65-103.

Biró et al. 1983-1995

K. Biró, J. Regenye, G. Ilon, E. BÁcskay, Angaben, Abhandlungen und Manuskripte publiziert in Zusammenhang mit dem Bergwerk Szentgál-Feuersteinberg und mit den Werkstattsiedlungen, http://www.ace.hu/szentgal/angab.html (letzter Zugriff 1.10.2019).

BRANDL 2013-2014

M. BRANDL, Genesis, provenance and classification of rocks within the chert group in central Europe, Archaeologia Austriaca 97-98, 2013-2014, 33-58.

BRANDL 2016

M. Brande, The Multi Layered Chert Sourcing Approach (MLA): analytical provenance studies of silicite raw materials, Archeometriai Múhely XIII/3, 2016, 145-156.

Brande, Trnka 2014

M. BRAndL, G. TRnkA, The eastern fringe: lithic raw materials from the easternmost Alps in Austria. In: Górnictwo z epoki kamienia: Krzemionki - Polska - Europa: w 90. rocznicę odkrycia kopalni w Krzemionkach. Silex et Ferrum 1, Ostrowiec Świętokrzyski 2014, 335-359.

Brandl, Penz, Schmitsberger im Druck

M. Brandl, M. Penz, O. Schmitsberger, KG Ober St. Veit, 13. Bezirk, Grabung Gemeindeberg, Fundberichte aus Österreich 57/2018, im Druck.

Brandl, Schmitsberger, Trnka 2018

M. Brandl, O. Schmitsberger, G. Trnka, News from the eastern fringe - the Baunzen Site near Vienna (Austria). In: D. H. Werra, M. Woźny (Hrsg.), Between History and Archaeology. Papers in honor of Jacek Lech. Oxford 2018, 59-68.

BRANDL et al. 2014

M. Brandl, C. Hauzenberger, W. Postl, M. M. Martinez, P. Filzmoser, G. Trnka, Radiolarite studies at Krems-Wachtberg (Lower Austria): Northern Alpine versus Carpathian lithic resources, Quaternary International 351, 2014, 146-162.

Dollinger, Wenty 1962

E. Dollinger, K. Wenty, Steinzeitlicher Bergbau auf dem Wiener Boden, Österreichischer Berg- und Hüttenkalender, 1962, 84-89.

Felgenhauer 1967

F. FelgenhaueR, Wien XXIII. - Kalksburg, Fundberichte aus Österreich 6/1951-1955, 1967, 27.

Felgenhauer, Mossler 1969

F. Felgenhauer, G. Mossler, Wien 13, Titlgasse, Fundberichte aus Österreich 9/4/1966-1970, 1969, 168.

GRILlO 1997

A. GRILlo, Hornsteinnutzung \& -handel im Neolithikum Südostbayerns. Beiträge zur Ur- und Frühgeschichte Mitteleuropas 12, Weißbach 1997.

Oliva 2010

M. Oliva, Pravěké hornictví v Krumlovském lese: Vznik a vývoj industriálně-sakrální krajiny najižní Moravě / Prehistoric Mining in the 'Krumlovský les' (Southern Moravia): Origin and Developement of an Industrial-Sacred Landscape. Anthropos $32=$ N.S. 24, Brünn 2010. 
Penz 2007

M. Penz, Die Bedeutung des Gemeindeberges in Wien 13, Ober St. Veit als jungsteinzeitlicher Siedlungsplatz, Fundort Wien 10, 2007, 194-197.

Penz 2016

M. PenZ, Wien 13, Lainzer Tiergarten - Inzersdorfer Wald und Faßlberg, Fundort Wien 19, 2016, 186.

Penz, Reiter 2012

M. Penz, V. Reiter, Wiener Fundstücke in der Studiensammlung des Institutes für Ur- und Frühgeschichte der Universität Wien, Fundort Wien 15, 2012, 152-171.

Penz, Schmitsberger 2016

M. Penz, O. Schmitsberger, Eine neu entdeckte (neolithische?) Hornsteinhalde im Lainzer Tiergarten/Inzersdorfer Wald in Wien, Fundort Wien 19, 2016, 144-147.

Pfersmann 2013

C. Pfersmann, Stratigraphy, Lithofacies and Geochemistry of the St. Veit Klippenzone and the Flysch Units from the Lainz Tunnel. Dissertation, Universität Wien 2013.

PREY 1979

S. PREY, Der Bau der Hauptklippenzone und der Kahlenberger Decke im Raume Purkersdorf-Wienerwaldsee (Wienerwald), Verhandlungen der Geologischen Bundesanstalt 2-1979, 1979, 205-228.

PREY 1991

S. Prey, Zur tektonischen Position der Klippe der Antonshöhe bei Mauer - Eine Richtigstellung, Jahrbuch der Geologischen Bundesanstalt 134/4, 1991, 845-847.

Ruiz-Ortiz, Bustillo, Molia 1989

P. A. Ruiz-Ortiz, M. A. Bustillo, J. M. Molia, Radiolarite sequences of the Subbetic, Betic Cordillera, Southern Spain. In: J. R. Hein, J. A. Obradović (Hrsg.), Siliceous Deposits of the Tethys and Pacific Regions. New York 1989, 111-113.

RUTTKAY 1970

E. RutTkay, Das jungsteinzeitliche Hornsteinbergwerk mit Bestattung von der Antonshöhe bei Mauer (Wien 23) - Die Ausgrabungen Josef Bayers in den Jahren 1929-1930, Mitteilungen der Anthropologischen Gesellschaft in Wien 100, 1970, 70-115.

SCHMITSBERGER 2015

O. Schmitsberger, KG Auhof, 13. Bezirk, KG Ober St. Veit, 13. Bezirk [Hörndlwald], Fundberichte aus Österreich 54, 2015, 443.

SCHMITSBERGER 2019a

O. Schmitsberger, KG Auhof, 13. Bezirk [Vösendorfergraben], Fundberichte aus Österreich 56/2017, 2019, 545.

SCHMitSBERGER 2019b

O. Schmitsberger, KG Ober St. Veit, 13. Bezirk [Gemeindeberg], Fundberichte aus Österreich 56/2017, 2019, 545.

SCHMITSBERgER im Druck a

O. Schmitsberger, BergbauLandschaftWien - Ein Prospektionsprojekt zum prähistorischen Hornsteinabbau in Wien 2017 und 2018, Fundberichte aus Österreich 57/2018, im Druck.

SCHMitSBERgER im Druck b

O. Schmitsberger, Bericht Teil A zum Prospektionsprojekt BergbauLandschaftWien, Fundberichte aus Österreich 57/2018, im Druck.

Schmitsberger im Druck c

O. Schmitsberger, Jungsteinzeitlicher Radiolaritabbau am Gemeindeberg in Wien: Bericht Teil B zur Grabung 2018, Fundberichte aus Österreich 57/2018, im Druck.
Schmitsberger, Neugebauer-Maresch 2015

O. Schmitsberger, C. Neugebauer-Maresch, Prospektion Paläolithikum Stadtgebiet Wien, Fundberichte aus Österreich 54, 2015, D7492-D7494.

Schmitsberger, Neugebauer-Maresch 2018a

O. Schmitsberger, C. Neugebauer-Maresch, KG Grinzing u. a., 19. Bezirk u. a. [Projekt-Endbericht „Gog \& Magog“], Fundberichte aus Österreich 55/2016, 2018, 572-575.

Schmitsberger, Neugebauer-Maresch 2018b

O. Schmitsberger, C. Neugebauer-Maresch, „Gog \& Magog die Mammutjägerzeit in Wien “, Prospektionsprojekt zum Paläolithikum 2015-2016, Fundberichte aus Österreich 55/2016, 2018, D8614-D8626.

Schmitsberger, Neugebauer-Maresch 2018c

O. Schmitsberger, C. Neugebauer-Maresch, Rammkernsondierung Titlgasse, Prospektionsprojekt zum Paläolithikum 2016, Fundberichte aus Österreich 55/2016, 2018, D8763-D8767.

Schmitsberger, Neugebauer-Maresch 2019

O. Schmitsberger, C. Neugebauer-Maresch, KG Auhof, 13. Bezirk, KG Grinzing, 19. Bezirk [Bericht zum ProspektionsProjekt BergbauLandschaftWien], Fundberichte aus Österreich 56/2017, 2019, 521-523.

Schmitsberger, Penz 2017a

O. Schmitsberger, M. Penz, Zwei weitere neu entdeckte prähistorische Radiolarit-Abbaustellen bzw. Schlagabfallhalden im Lainzer Tiergarten in Wien, Fundort Wien 20, 2017, 152-161.

Schmitsberger, Penz 2017b

O. Schmitsberger, M. Penz, Wien 13, Lainzer Tiergarten - Dorotheer Wald und Untere Wildpretwiese, Fundort Wien 20, 2017, 197.

Schmitsberger, Penz 2018a

O. Schmitsberger, M. Penz, Klippen, Bergbau, Schlagabfällle Neu entdeckte Radiolarit-Abbaustellen im Lainzer Tiergarten in Wien mit einem ersten Überblick über die Fundstellen in der „Bergbauzone Tiergarten“, Fundort Wien 21, 2018, 120-146.

Schmitsberger, Penz 2018b

O. Schmitsberger, M. Penz, Wien 13 - Lainzer Tiergarten (Südostbereich) - Dorotheer und Inzersdorfer Wald, Fundort Wien 21, 2018, 222.

Schmitsberger, Penz im Druck a

O. Schmitsberger, M. Penz, Die Radiolarit-Artefaktfundstelle „Hörndlwald“ im 13. Wiener Gemeindebezirk - ein Beitrag zur neolithischen „Bergbau-Infrastruktur“ im Umfeld der St. Veiter Klippenzone, Fundberichte aus Österreich 57/2018, im Druck.

Schmitsberger, Penz im Druck b

O. Schmitsberger, M. Penz, Hornstein, Halden, Hammersteine - Vierter Vorbericht zur Prospektion im Lainzer Tiergarten im Rahmen des Projekts „BergbauLandschaftWien“, Fundort Wien 22, im Druck.

Schmitsberger, Penz im Druck c

O. Schmitsberger, M. Penz, KG Ober St. Veit, 13. Bezirk [Fundbericht Roter Berg], Fundberichte aus Österreich 57/2018, im Druck.

ŚLĄCZKA et al. 2018

A. Ślączka, M. Bąk, C. Pfersmann, V. Koukal, M. Wagreich, S. Kowalik, M. Maslo, Jurassic-Cretaceous radiolarian-bearing strata from the Gresten Klippen Zone and the St. Veit Klippen Zone (Wienerwald, Eastern Alps, Austria): Implications for stratigraphy and paleogeography, Austrian Journal of Earth Sciences 111/2, 2018, 204-222. 
SCHNABEL 1998

W. Schnabel (Bearb.), Geologische Karte der Republik Österreich 1:50.000, Blatt 58: Baden. Wien 1998.

Trauth 1930

F. Trauth, Geologie der Klippenregion von Ober-St. Veit und des Lainzer Tiergartens, Mitteilungen der Geologischen Gesellschaft in Wien 21/1928, 1930, 35-132.

TRNKA 2014

G. TRNKA, The Neolithic radiolarite mining site of Wien-Mauer-Antonshöhe (Austria). In: K. T. Bıró, A. Markó, K. P. BAJnoK (Hrsg.), Aeolian Scripts: New Ideas on the Lithic World. Studies in Honour of Viola T. Dobosi. Inventaria Praehistorica Hungariae 13, Budapest 2014, 235-245.

Oliver Schmitsberger Institut für Orientalische und Europäische Archäologie Österreichische Akademie der Wissenschaften Hollandstraße 11-13 1020 Wien Österreich oliver.schmitsberger@oeaw.ac.at (iD) orcid.org/0000-0002-0553-1498

Michael Brandl Institut für Orientalische und Europäische Archäologie Österreichische Akademie der Wissenschaften Hollandstraße 11-13 1020 Wien

Österreich michael.brandl@oeaw.ac.at orcid.org/0000-0002-0214-8231

Martin Penz Museen der Stadt Wien - Stadtarchäologie Obere Augartenstraße 26-28 1020 Wien Österreich martin.penz@stadtarchaeologie.at (iD) orcid.org/0000-0002-1895-8044 